\title{
TREATMENT OF BLUNDER BUSS CANAL: A CASE REPORT
}

Dileep Soni' ${ }^{1}$, Deepak Raisingani², Rachit Mathur ${ }^{3}$, Neha Mehta ${ }^{4}$, Ritu Meel ${ }^{5}$

\section{HOW TO CITE THIS ARTICLE:}

Dileep Soni, Deepak Raisingani, Rachit Mathur, Neha Mehta, Ritu Meel. "Treatment of Blunder Buss Canal: A Case Report". Journal of Evolution of Medical and Dental Sciences 2014; Vol. 3, Issue 06, February 10; Page: 1440-1447, DOI: $10.14260 /$ jemds/2014/2004

\begin{abstract}
The aim of this case report was to evaluate the depth of cure of composite resin cured within root canals by means of light-transmitting plastic posts. Light-curing composite resins must be adequately polymerized within the root canal to reinforce weakened roots. The physical properties of dental composite resins are of great concern because they affect their clinical performance. The lighttransmitting plastic posts (Luminex, Weissman Technology) have enabled the transmission of light into the root canal to polymerize composite resin placed within it.
\end{abstract}

KEYWORDS: Blunderbuss canal, MTA, light transmission, light transmission post.

INTRODUCTION: The flared canal arises as a result of carious extension, trauma to an immature tooth pulpal pathosis. Iatrogenic or endodontic misadventure, or idiopathic causes, can present a difficult restorative problem to the practicing dentist or endodontist. Placement of retentive pins is impossible because of the lack of dentinal structure at the coronal portion of the root. The use of conventional tapered, cast posts would concentrate wedging forces, further stressing the critically weakened coronal end of the post canal. The introduction of materials capable of bonding to dentinal structure has created potential for the reconstitution and rehabilitation of lost dentinal tissues to salvage severely damaged teeth that would otherwise be extracted. When the weakened root is internally rebuilt with suitable adhesive dental materials, the root is dimensionally and structurally reinforced to support and retain a post and core for continued function of the tooth. ${ }^{1}$

CASE REPORT: A30-year-old male presented to the Department of Conservative Dentistry \& Endodontics, with a chief complaint of spontaneous toothache in maxillary right central incisor for the past five months. The patient's medical history was unremarkable. The tooth was sensitive to temperature variations and electric pulp test and tender to vertical percussion. The pre-operative radiograph on maxillary right central incisor (Figure 1) shows a large deep coronal root defect caused by gross intra-radicular extension of caries into a previously traumatized central incisor. Preoperative radiograph showed coronal radiolucency involving pulpal radiolucency (with open apex -blunderbuss canal) with large periapical radiolucency indicating chronic peri-apical abscess.

Local anesthesia was administered with 2\% lidocaine and 1:80000 epinephrine and a rubber dam was placed. After removal of caries the pulp chamber was completely rinsed with normal saline. Working length radiograph was taken by use of \# 80 no. K file (due to the presence of large blunderbuss canal) (Figure-2). The $4 \mathrm{~mm}$ of apical portion of the canal was closed with orthograde MTA placement and wet cotton pellet was place over the MTA plug which was then sealed with cavit (Figure-3). Patient was recalled next day then the wet cotton was removed to fit a correspondingsized light-transmitting plastic post carrying a depth-marking ring; according to the desired size and depth of canal. The matched post is removed and the internal root dentin is acid etched (eg. Tooth Conditioner Gel, Caulk/Dentsply) rinsed, and dried. To bond the composite resin to dentin, a dentinal 
bonding system (eg, Prisma Universal Bond 3, Caulk/Dentsply) is used according to the manufacturer's instructions. The dentinal primer is applied with a brush over the dentinal surfaces and dried with oil-free air. The adhesive is then similarly applied over the primed dentin. After the light-transmitting post is reinserted, the adhesive is cured for 10 seconds with a suitable light-curing unit. The light-transmitting post is again removed. Visible light-curing hybrid composite resin (eg, Prisma AP, H, Caulk/Dentsply) is selected, its placement into the depths of the root canal (Figure4).The light-transmitting post is reseated to its full depth to ensure that the desired post canal length is achieved clinically (Figure-5) and at the same time, through the pressure exerted, to facilitate good adaptation of the composite resin against the canal walls. Following removal of excess material from the coronal root face, a light-curing unit is applied to the end of the plastic post to transilluminate light along its entire length to polymerize the surrounding composite resin. The light-transmitting post can be cemented into the prepared canal radiographically (Figure-6) and a composite resin core can be built up clinically (Figure-7). Finally, the clinical technique was completed with the cementation of an esthetic porcelain crown \{radiograph and clinical image (Figure 8, 9, 10)\}.

\section{DISCUSSION:}

Clinical Applications: There are many clinical situations in which an internally damaged root can be endodontically treated and rehabilitated in conjunction with preparation of the post canal. Generally in these cases, the defect assumes a flared configuration at the coronal portion of the root canal, while the apical portion has adequate dentinal support. The entire external root surface is also essentially intact and adequately supported by periodontal tissues. ${ }^{2}$ It is therefore fundamentally sound to reconstitute the weakened coronal portion so that the rehabilitated root is rendered capable of supporting a restoration and thereby continuing the usefulness of the tooth. This technique is especially recommended for the reinforcement and rehabilitation of function and esthetics to anterior teeth in an otherwise intact arch, as in the following instances:

1. Extension of caries into the coronal portion of the root canal.

2. Trauma to immature incisors.

3. Developmental anomalies, such as fusion and germination.

4. Idiopathic pulpal pathoses, such as internal résorption.

5. Iatrogenic damage, such as excessive preparation of an access cavity, excessive taper of a post canal preparation, or other restorative or endodontic misadventure..$^{3,4}$

The depth of cure of visible light-curing composite resin achieved at best in the conventional manner is 2 to $3 \mathrm{~mm}$, because of the limited transillumination of light through the composite resin. With the introduction of light-transmitting plastic posts, it is possible to transilluminate light through the bulk of deeper, intra-radicularly placed composite resin, because light is transmitted along the entire length of the plastic post. ${ }^{5}$ Complete polymerization of the composite resin along the entire length and circumference of the flared root canal is thus possible. Following polymerization and removal of the plastic post, a patent, accurate, and retentive post canal is immediately established for the intended matching post. 6

This technique thereby ensures that retention and resistance requirements of post and core systems can be conveniently and easily met as the reconstituted post canal can accommodate a passive, parallel sided, well-fitting post. For a flared canal, the use of a cast post can concentrate 
wedging forces at the weakened coronal portion of the root. The use of a prefabricated post, however, entails the obturation of the large defect with a cementing medium a very weak area in the entire post-core-crown-tooth complex. Therefore, it is practical to reinforce this weakened intra-radicular portion by rebuilding the lost dentin with a strong dentinal substitute. ${ }^{7}$

Many clinicians have advocated the use of composite resin as a reinforcing material. Good bonding of composite resins to dentin is now possible because of the advances in dentinal adhesives, in a flared canal, the composite resin bonded to the root dentinal surfaces would dimensionally and structurally reconstitute the defective portion, thereby reinforcing the weakened root. ${ }^{8,9}$ Use of autocuring composite and light-curing composite resins in the conventional manner can give rise to problems in the depths of the root canal. ${ }^{3}$

The use of light-transmitting posts in conjunction with light-curing composite resins within a post canal space eliminates the difficulty in control experienced with a rapidly polymerizing autocuring composite resin and ensures complete polymerization in a light curing composite resin. Besides internally rehabilitating and rebuilding the weakened root, the light-transmitting plastic post at the same time form an optimal post canal. ${ }^{4}$ Because posts are intended to provide retention and resistance to displacement of the core, the post canal should be as small as the apical dentin portion of the root canal would fit the smallest acceptable size post.

This would ensure that the diametric dimension of the composite resin reinforcement is effectively increased to better resist fracture to the root, In this respect six sizes of light-transmitting plastic posts, varying from 1,05 to $1,80 \mathrm{~mm}$ and with a $0,15-\mathrm{mm}$ difference between sizes, are available from the manufacturer. ${ }^{5}$ A tooth with a flared canal in an otherwise intact arch presents the dentist with a restorative problem. Previously, such a tooth, even in the maxillary anterior region, would be deemed unrestorable and would usually be extracted. ${ }^{6}$

Today, with rapid advancement in adhesive techniques and materials, restorative dentistry has shifted to conservation of even badly damaged teeth and their restoration to function and esthetics so as to best serve the needs of the patient. Through reconstitution of the intra-radicular defect, the compromised tooth is reinforced and rehabilitated to retain a post and core and support a function of esthetic crown.

The reconstitution and reinforcement can be easily and successfully achieved by using lightcuring composite resins polymerized with the aid of light-transmitting plastic posts. Flared canals resulting from damage caused by caries, trauma, congenital disorder, internal résorption, or iatrogenic or other idiopathic causes can thus be adequately reinforced by using the technique described. 10

SUMMARY: The introduction of light-transmitting plastic posts has enabled the use of light-curing composite resin for intra-radicular reinforcement of flared canals in weakened endodontically treated roots. This simple clinical technique allows better control of the reinforcing resin during its placement and ensures more complete polymerization of the light-curing composite resin within the depths of the root canal. The plastic post also allows the dentist to obtain an accurate post canal that can optimally accommodate a matching, passive, parallel sided post and provide the necessary retention and maximum resistance to displacement. The use of light transmitting plastic posts in combination with adhesive materials is in keeping with a conservative rehabilitative technique for the restoration of badly damaged teeth. 


\section{REFERENCES:}

1. Anantharaj et al. Challenges in pulpal treatment of young permanent teeth a review. Journal of Dental Sciences and Research: 2011: Volume 2: Issue 1: February.

2. Richard D. Trushkowsky. Aesthetic and Functional Consideration in Restoring Endodontically Treated Teeth. Dent Clin N Am 2011: 55:403-410.

3. Oguz Yoldas and Tayfun Alaçam. Microhardness of Composites in Simulated Root Canals Cured with Light Transmitting Posts and Glass-Fiber Reinforced Composite Posts. JOE2005: Volume 31, Number 2, February.

4. Richard S. Schwartz and James W. Robbins. Post Placement and Restoration of Endodontically Treated Teeth: A Literature Review. JOE 2004: Volume 30, Number 5: May.

5. André Luís Faria e Silva. Influence of Fiber-post Translucency on the Degree of Conversion of a Dual-cured Resin Cement. JOE2007: Volume 33, Number 3, March.

6. Cleonice Silveira Teixeira, Yara T. Corre^a Silva-Sousa and Manoel D. Sousa-Neto. Bond Strength of Fiber Posts to Weakened Roots after Resin Restoration with Different Light-Curing Times. JOE2009: Volume 35, Number 7, July.

7. Akkayan B, Gulmez T. Resistance to fracture of endodontically treated teeth restored with different post systems. J Prosthet Dent 2002; 87:431-7.

8. Cormier CJ, Burns DR, Moon P. In vitro comparison of the fracture resistance and failure mode of fiber, ceramic, and conventional post systems at various stages of restoration. J Prosthodont 2001;10:26-36.

9. Newman MP, Yaman P, Dennison J, Rafter M, Billy E. Fracture resistance of endodontically treated teeth restored with composite posts. J Prosthet Dent 2003;89:360-7.

10. Luís Fernando dos Santos Alves Morgan, DDS; Light Transmission through a Translucent Fiber Post; JOE2008: Volume 34, Number 3, March.

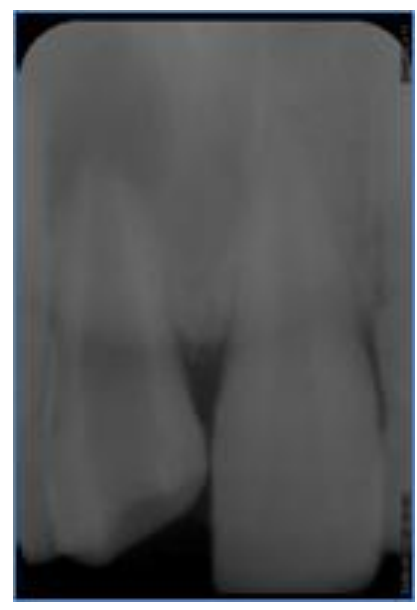

Fig. 1: Intraoral Peri-apical radiograph showed large defect of the maxillary right central incisor caused by carious extension into a previously traumatized, immature incisor. 


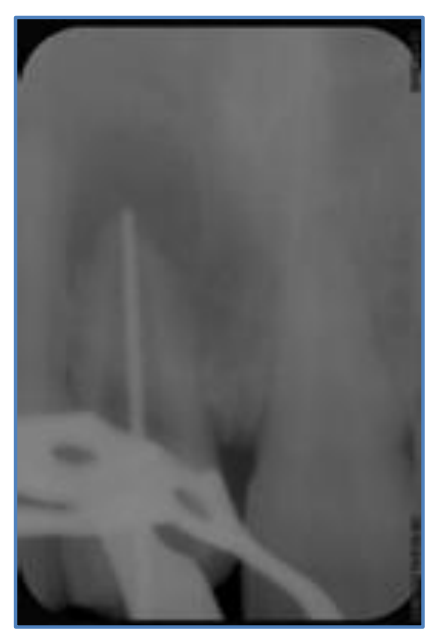

Fig. 2: Working length in

intraoral Peri-apical radiograph

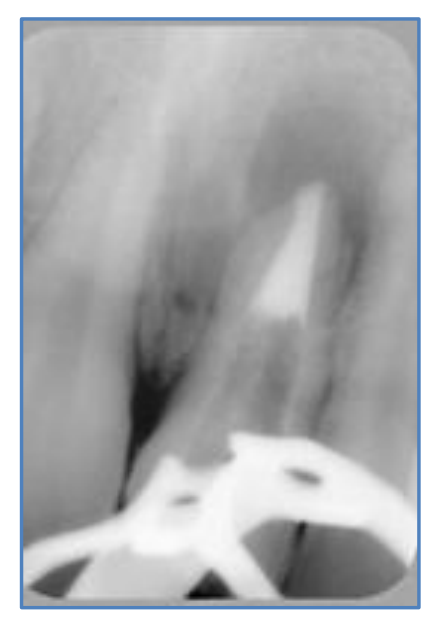

Fig. 3: $4 \mathrm{~mm}$ of orthograde MTA placed

into the prepared root canal central incisor

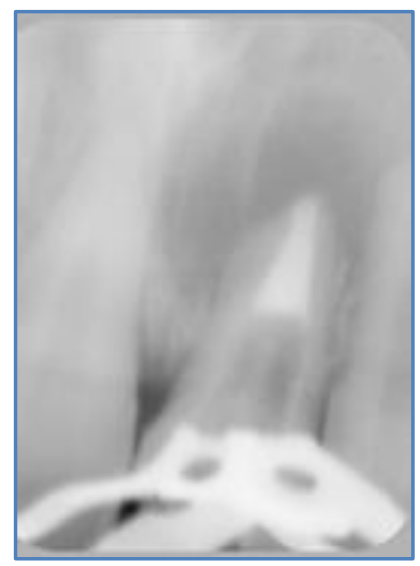

Fig. 4: A matched light-transmitting plastic post is fitted into the prepared root canal central incisor (Intra-Oral Peri-apical Radiograph) 


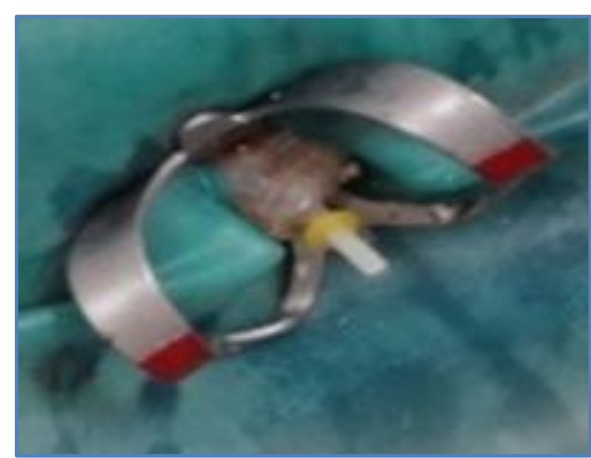

Fig. 5: A matched light-transmitting plastic post is fitted into the prepared root canal central incisor (Intra-Oral Image)

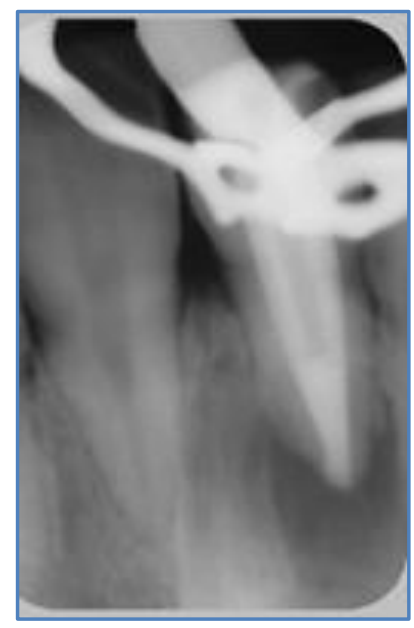

Fig. 6: Placement of composite resin into the bonded light transmitting plastic post root canal

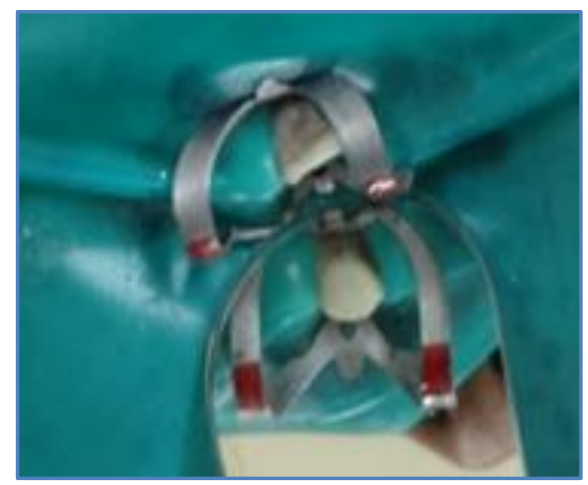

Fig. 7: A composite resin core is built up and secured onto cross out head of the prefabricated cemented post. 


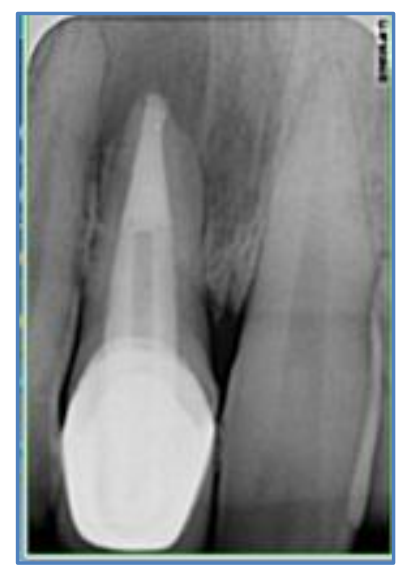
Fig. 8: The reinforced central incisor is finally restored to esthetics and function with a porcelain fussed metal crown.

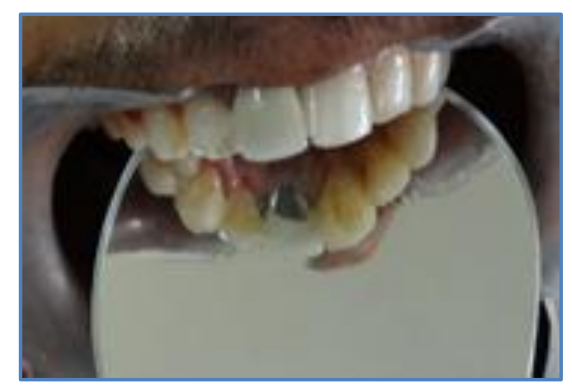

Fig. 9: The reinforced central incisor is finally restored to esthetics and function, with a porcelain fused metal crown. (Intra-Oral Image)
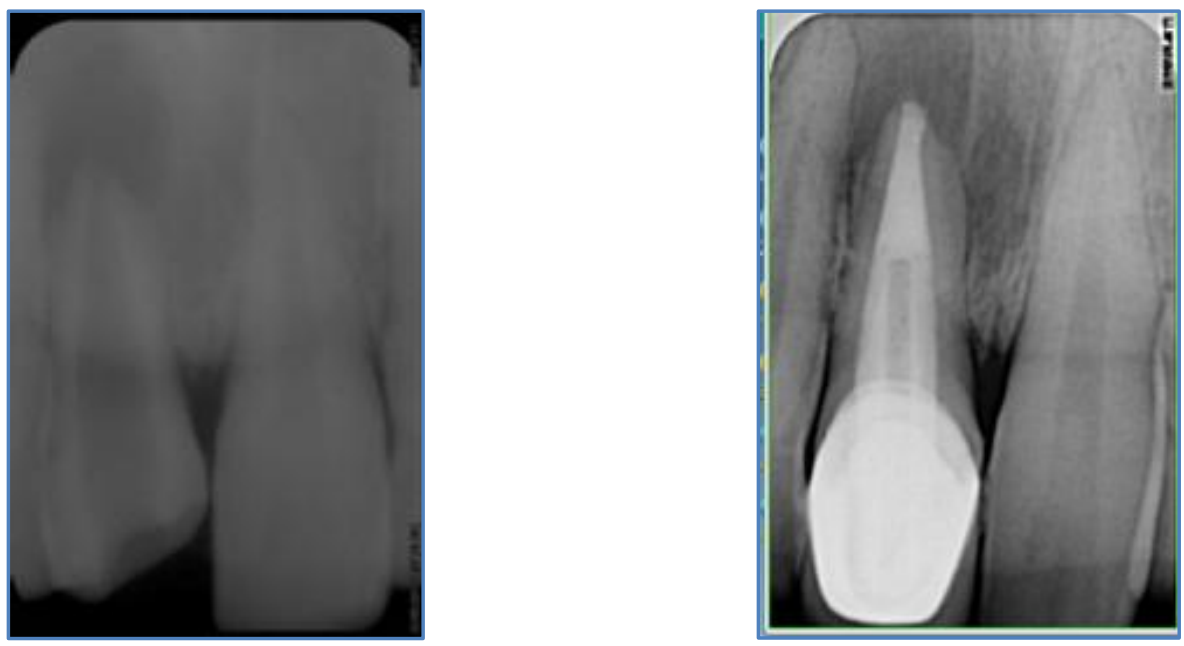

Fig. 10: Clinical Technique is completed with the cementation (before and after radiographically) 


\section{CASE REPORT}

\section{AUTHORS:}

1. Dileep Soni

2. Deepak Raisingani

3. Rachit Mathur

4. Neha Mehta

5. Ritu Meel

\section{PARTICULARS OF CONTRIBUTORS:}

1. Post-Graduate, Department of Conservative Dentistry and Endodontics, Mahatma Gandhi Dental College and Hospital, Jaipur.

2. Professor and HOD, Department of Conservative Dentistry and Endodontics, Mahatma Gandhi Dental College and Hospital, Jaipur.

3. Senior Lecturer, Department of Conservative Dentistry and Endodontics, Mahatma Gandhi Dental College and Hospital, Jaipur.
4. Post-Graduate, Department of Conservative Dentistry and Endodontics, Mahatma Gandhi Dental College and Hospital, Jaipur.

5. Post Graduate, Department of Conservative Dentistry and Endodontics, Mahatma Gandhi Dental College and Hospital, Jaipur.

\section{NAME ADDRESS EMAIL ID OF THE CORRESPONDING AUTHOR:}

Dr. Dileep Soni,

Post Graduate, Department of Conservative Dentistry and Endodontics,

Mahatma Gandhi Dental College \& Hospital, Jaipur - 302022.

E-mail: dileeo01.soni@gmail.com

Date of Submission: 22/01/2014.

Date of Peer Review: 23/01/2014.

Date of Acceptance: 30/01/2014.

Date of Publishing: 05/02/2014. 